\title{
Association of nursing-documented ambulation with length of stay following total laparoscopic hysterectomy for benign gynecologic disease
}

\author{
Kidong Kim', Sooyoung Yoo ${ }^{2}$, Eun Joo Yang ${ }^{3}$, Jae Hong No ${ }^{1}$, Hee Hwang ${ }^{2,4}$, Yong-Beom Kim \\ ${ }^{1}$ Department of Obstetrics and Gynecology, ${ }^{2}$ Center for Medical Informatics, ${ }^{3}$ Department of Rehabilitation Medicine, ${ }^{4}$ Department of Pediatrics, Seoul \\ National University Bundang Hospital, Seoul National University College of Medicine, Seongnam, Korea
}

\section{Objective}

The objective was to examine the association of postoperative physical activity with length of stay in patients who received total laparoscopic hysterectomy for benign gynecologic disease.

\section{Methods}

The case group was composed of 70 patients who entered a critical pathway for elective total laparoscopic hysterectomy from 2009 to 2012 and were discharged behind schedule. The control group was selected from patients who were discharged on schedule, and matched to cases using 1:3 ratio propensity score matching. We compared the number of nursing-documented ambulation of the case group with that of control group.

Results

Year of surgery, age, body mass index, endometriosis, systemic disease, previous abdominal surgery and current medication were well balanced between case and control groups. The number of patients with nursing-documented ambulation in case group (19\%) was not different from that in control group (11\%).

\section{Conclusion}

Postoperative physical activity measured by nursing-documented ambulation was not associated with length of stay in patients who underwent an elective total laparoscopic hysterectomy for benign gynecologic diseases.

Keywords: Ambulation; Hysterectomy; Length of stay; Nursing records; Postoperative care

\section{Introduction}

Postoperative physical inactivity is a risk factor for various adverse outcomes. For example, a retrospective study reported that postoperative prolonged bed rest is a risk factor for pulmonary embolism following cardiac surgery [1]. A systematic review showed that immobilization defined as confinement to bed and/or armchair was associated with 6 fold increase in deep vein thrombosis [2]. A randomized trial including patients who underwent a coronary artery bypass surgery revealed that systematic turning activity in bed decreased the risk of postoperative fever and length of stay (LOS) in intensive care unit [3]. A retrospective study found that delayed ambulation after hip fracture surgery was related to delirium, pneumonia and increased LOS [4].
LOS is one of the most important metrics for cost-effective care [5]. In addition, adequate LOS was associated with

Received: 2013.2.25. Revised: 2013.4.6. Accepted: 2013.4.13.

Corresponding author: Yong-Beom Kim

Department of Obstetrics and Gynecology, Seoul National University Bundang Hospital, Seoul National University College of Medicine, 82 Gumi-ro 173beon-gil, Bundang-gu, Seongnam 463-707, Korea Tel: +82-31-787-7253 Fax: +82-31-787-4054

E-mail: ybkimlh@snubh.org

Articles published in Obstet Gynecol Sci are open-access, distributed under the terms of the Creative Commons Attribution Non-Commercial License (http://creativecommons. org/licenses/by-nc/3.0/) which permits unrestricted non-commercial use, distribution, and reproduction in any medium, provided the original work is properly cited.

Copyright $\odot 2013$ Korean Society of Obstetrics and Gynecology 


\section{Obstetrics \& Gynecology Science}

Kidong Kim, et al. Physical activity and length of stay

quality of care in several settings. For example, a nationwide population-based cohort study showed that higher volume hospitals significantly reduced LOS and in-hospital mortality for elderly patients with acute biliary diseases [6]. Another study reported that patients admitted to high volume stroke units received a higher quality care and spent fewer days in the hospital [7].

The association of physical activity during hospitalization with LOS was mainly investigated in elderly populations. For example, an observational cohort study examined the total number of steps in elderly patients hospitalized with acute medical illness and reported that patients with shorter LOS tended to have higher physical activity [8]. A possible association of physical activity with LOS following surgery can be deduced from trials testing the benefit of fast-track approach. Specifically, the fast-track approach including early ambulation after surgery was known to reduce LOS in various settings [9-11]. However, in trials testing the fast-track approach, the physical activity of patients was not measured. Therefore, to our knowledge, the association of physical activity during hospitalization with LOS was not thoroughly investigated in patients following surgery.

The objective of this study was to examine the association of postoperative physical activity measured by nursingdocumented ambulation with LOS following an elective total laparoscopic hysterectomy for benign gynecologic diseases.

\section{Materials and methods}

\section{Population}

From 2009 to 2012, 745 patients with benign gynecologic diseases entered a critical pathway for elective total laparoscopic hysterectomy in our institute. All patients received same perioperative management according to a prespecified protocol. Patients were admitted the day before operation day and received a bowel preparation using bisacodyl rectally and laxatives orally and rectally. On operation day, prophylactic antibiotics were intravenously given 30 minutes before skin incision and no postoperative antibiotics were administered. On postoperative first day, diet was tried and ambulation was encouraged. On postoperative second day, patients were discharged. Postoperative pain was managed with ketorolac tromethamine intravenously administered via patient-controlled analgesia device.
After the approval of Institutional Review Board, the characteristics of 745 patients were abstracted from medical records. One hundred twenty-five patients with intraoperative severe complications, concurrent surgeries or unexpected cancer diagnosis were excluded. For the remaining 620 patients, age, body mass index, endometriosis, systemic disease, previous abdominal surgery and current medication were abstracted from medical records. Seventy patients who were discharged on postoperative third day or later comprised the case group and the other 550 patients were the initial control group.

The Institutional Review Board reviewed this protocol in a brief review track and exempted this study from further review.

\section{Matching}

Propensity score calculation and matching were performed using Matchlt package in R ver. 2.15.0 (R Foundation for Statistical Computing, http://www.R-project.org) [12]. Year, age, body mass index, endometriosis, systemic disease, previous abdominal surgery and current medication were used to calculate the propensity score. The ratio for case and control was $1: 3$ and the nearest matching was performed. Of 550 patients in initial control group, 210 patients were matched and composed a matched control group.

\section{Measurement of physical activity}

In our institution, nurses described the status of patients using the predetermined sentences in electronic medical record. There were nine sentences describing the ambulation event of patients. For example, the sentence of "The patient is ambulating" is used to describe the ambulation event of patients. We examined whether the sentences describing the ambulation event was present in nursing record of each patient from operation to $9 \mathrm{AM}$ of postoperative second day. When the sentences describing the ambulation event was present one or more times, the patient was determined to have a nursingdocumented ambulation.

\section{Analysis}

Characteristics of the case group were compared with those of the initial and matched control group. Chi-square or Fisher's exact test was used for categorical variables. Student's $t$ test was used for continuous variables. If there is a significant difference in any variable between case and matched control groups, ratio for matching was planned to be reduced. When 


\title{
Obstetrics \& Gynecology Science
}

\author{
Vol. 56, No. 4, 2013
}

the difference was not corrected despite reduction of ratio, conditional logistic regression analysis including the variable with difference was planned. Propensity score calculation and matching were performed using Matchlt package in $\mathrm{R}$ ver. 2.15.0 and the other analyses were performed using SPSS ver. 20.0.0 (SPSS Inc., IBM Co., Somers, NY, USA). Null hypotheses of no difference were rejected if $P$-values were less than 0.05 .

\section{Results}

\section{Baseline characteristics}

The characteristics of case, matched control, and initial control groups are summarized in Table 1. Over $70 \%$ of patients received an operation in 2010 and 2011. The mean age of the cases was 48 years, of the matched controls 48 and the initial control group 47 . The mean body mass index was $24 \mathrm{~kg} / \mathrm{m}^{2}$ in all groups. The prevalence of endometriosis, systemic disease, previous abdominal surgery and current medication was not different among groups, and all variables were well-balanced between case and matched control groups.

\section{Physical activity}

The number of patients with nursing-documented ambulation in case group (19\%) was not different from that in matchedcontrol group (11\%) (Table 2).

\section{Discussion}

\section{Our finding}

In this study, nursing-documented ambulation was not associated with LOS. Several explanations are possible. First, patients who underwent total laparoscopic hysterectomy for benign gynecologic diseases might not be an ideal group to test the hypothesis of 'physical activity of patients with longer LOS would be less than that of patients with shorter LOS.' In other words, most patients who underwent total laparoscopic hysterectomy would not experience significant decline of function resulting in reduced physical activity and longer LOS. Second, nursing-documented ambulation might not be a reliable method to measure physical activity. Nursing-documented ambulation could be affected by various factors such as

Table 1. The characteristics of case, matched control, and initial control groups

\begin{tabular}{|lccc|}
\hline Characteristic & $\begin{array}{c}\text { Case } \\
(\mathbf{n}=\mathbf{7 0})\end{array}$ & $\begin{array}{c}\text { Matched control } \\
(\mathbf{n}=\mathbf{2 1 0})\end{array}$ & $\begin{array}{c}\text { Initial control } \\
(\mathbf{n}=550)\end{array}$ \\
\hline Year & & & $33(6)$ \\
2009 & $8(11)$ & $12(6)$ & $257(47)$ \\
2010 & $22(31)$ & $71(34)$ & $204(37)$ \\
2011 & $28(40)$ & $95(45)$ & $56(10)$ \\
2012 & $12(17)$ & $32(15)$ & $47 \pm 8$ \\
Age (yr) & $48 \pm 8$ & $48 \pm 8$ & $24 \pm 3$ \\
Body mass index $\left(\mathrm{kg} / \mathrm{m}^{2}\right)$ & $24 \pm 3$ & $24 \pm 4$ & $28(5)$ \\
Endometriosis & $8(11)$ & $25(12)$ & $93(17)$ \\
Systemic disease & $9(13)$ & $30(14)$ & $249(45)$ \\
Previous abdominal surgery & $24(34)$ & $80(38)$ & $58(11)$ \\
Current medication & $3(4)$ & $12(6)$ & \\
\hline
\end{tabular}

Values are presented as number (\%) or mean \pm standard deviation.

Table 2. Association of nursing-documented ambulation with delay of discharge

\begin{tabular}{|lcc|}
\hline Nursing-documented ambulation & $\begin{array}{c}\text { Regular discharge } \\
(\mathbf{n}=\mathbf{2 1 0})\end{array}$ & $\begin{array}{c}\text { Delayed discharge } \\
(\mathbf{n}=\mathbf{7 0})\end{array}$ \\
\hline No & $187(89)$ & $57(81)$ \\
Yes & $23(11)$ & $13(19)$ \\
\hline
\end{tabular}

Values are presented as or number (\%).

$P>0.05$. 


\section{Obstetrics \& Gynecology Science}

Kidong Kim, et al. Physical activity and length of stay

walking time, walking area and nurse's attitude to document the ambulation. Recently, several studies used more accurate methods such as accelerometer or pedometer to measure the physical activity $[13,14]$.

\section{Low level of physical activity during hospitalization} In this study, only 36 of 280 patients (13\%) did a nursingdocumented ambulation. The low level of physical activity during hospitalization was reported in previous studies. For example, in a study examining the frequency of hallway ambulation in hospitalized patients aged $\geq 55$, only 318 of $19,363(2 \%)$ patient-minutes were used in hallway walking [15]. Another study measuring the number of steps in hospitalized older adults reported that the average number of steps per day was only one tenth of community-living older adults [8].

\section{Electronic nursing record using predetermined sentences}

We can measure the physical activity of hospitalized patients by counting the number of predetermined sentences describing the ambulation event. We think that electronic nursing record using predetermined sentences could be a powerful tool in evaluating the status of patients in a group. For example, we can measure the quality of postoperative pain control by counting the number of sentences describing the pain experience. Hypothetically, the increased number could trigger us to improve the quality of postoperative pain control.

\section{Future studies}

Although we did not find any association of physical activity with LOS in this study, we obtained valuable lessons for future studies. First, to detect the effect of physical activity to LOS, the study population should be a group with diseases causing significant decline of function. Second, physical activity should be measured prospectively through reliable methods such as accelerometer or pedometer.

In spite of the negative result of this study, we still believe that physical activity is an essential metric to evaluate the function and health status of patients. Therefore, we hold that accurate measurement of physical activity can help predict the prognosis of patients in various settings. Furthermore, we believe that patient prognosis could be improved by increasing the physical activity of patients. Further studies investigating the association of physical activity with function and health status are warranted.

\section{Conflict of interest}

No potential conflict of interest relevant to this article was reported.

\section{Acknowledgments}

The authors thank the Medical Research Collaborating Center at Seoul National University Bundang Hospital for statistical analyses.

\section{References}

1. Gillinov AM, Davis EA, Alberg AJ, Rykiel M, Gardner TJ, Cameron DE. Pulmonary embolism in the cardiac surgical patient. Ann Thorac Surg 1992;53:988-91.

2. Samama MM, Dahl OE, Quinlan DJ, Mismetti P, Rosencher N. Quantification of risk factors for venous thromboembolism: a preliminary study for the development of a risk assessment tool. Haematologica 2003;88:1410-21.

3. Chulay M, Brown J, Summer W. Effect of postoperative immobilization after coronary artery bypass surgery. Crit Care Med 1982;10:176-9.

4. Kamel HK, Iqbal MA, Mogallapu R, Maas D, Hoffmann RG. Time to ambulation after hip fracture surgery: relation to hospitalization outcomes. J Gerontol A Biol Sci Med Sci 2003;58:1042-5.

5. Taheri PA, Butz DA, Greenfield LJ. Length of stay has minimal impact on the cost of hospital admission. J Am Coll Surg 2000;191:123-30.

6. Murata A, Matsuda S, Kuwabara K, Ichimiya Y, Matsuda Y, Kubo T, et al. Association between hospital volume and outcomes of elderly and non-elderly patients with acute biliary diseases: A national administrative database analysis. Geriatr Gerontol Int 2012.

7. Svendsen ML, Ehlers LH, Ingeman A, Johnsen SP. Higher stroke unit volume associated with improved quality of early stroke care and reduced length of stay. Stroke 2012;43:3041-5.

8. Fisher SR, Goodwin JS, Protas EJ, Kuo YF, Graham JE, Ot- 


\section{Obstetrics \& Gynecology Science}

Vol. 56, No. 4, 2013

tenbacher KJ, et al. Ambulatory activity of older adults hospitalized with acute medical illness. J Am Geriatr Soc 2011;59:91-5.

9. Magheli A, Knoll N, Lein M, Hinz S, Kempkensteffen C, Gralla O. Impact of fast-track postoperative care on intestinal function, pain, and length of hospital stay after laparoscopic radical prostatectomy. J Endourol 2011;25:1143-7.

10. Sidhu VS, Lancaster L, Elliott D, Brand AH. Implementation and audit of 'Fast-Track Surgery' in gynaecological oncology surgery. Aust N Z J Obstet Gynaecol 2012;52:371-6.

11. Vlug MS, Wind J, Hollmann MW, Ubbink DT, Cense HA, Engel $A F$, et al. Laparoscopy in combination with fast track multimodal management is the best perioperative strategy in patients undergoing colonic surgery: a randomized clinical trial (LAFA-study). Ann Surg 2011;254:868-75.

12. R Development Core Team. R: a language and environment for statistical computing. Vienna, Austria: The $\mathrm{R}$ Foundation for Statistical Computing; 2001.

13. Allet L, Knols RH, Shirato K, de Bruin ED. Wearable systems for monitoring mobility-related activities in chronic disease: a systematic review. Sensors (Basel) 2010;10:9026-52.

14. Corder K, Brage S, Ekelund U. Accelerometers and pedometers: methodology and clinical application. Curr Opin Clin Nutr Metab Care 2007;10:597-603.

15. Callen BL, Mahoney JE, Grieves CB, Wells TJ, Enloe M. Frequency of hallway ambulation by hospitalized older adults on medical units of an academic hospital. Geriatr Nurs 2004;25:212-7. 